\title{
Dynamics of Sensory and Motor Deficiency against the Background of Transcutaneous Electro Neuro Stimulation in the Therapy of Patients with Diabetic Distal Polyneuropathy
}

\author{
Al Zamil $\mathbf{M}^{* 1}$, Minenko IA ${ }^{2}$ and Kulikova $\mathrm{NG}^{3}$ \\ ${ }^{1}$ Department of Advanced training faculty for Health professionals, People's Friendship University of Russia, Russia \\ ${ }^{2}$ Department of Integrative medicine of Sechenov University, Russia
}

${ }^{3}$ Department of Physiotherapy of the Advanced Training, People's Friendship University of Russia, Russia

Received: May 31, 2018; Published: June 11, 2018

*Corresponding author: Al Zamil M, Department of Advanced training faculty for Health professionals, Russia

\section{Abstract}

Introduction: Transcutaneous electroneurostimulation has been used for a long time in the treatment of neuropathic pain syndrome in type 2 diabetes mellitus. However, the effectiveness of this method in treating other manifestations of the distal polyneuropathy of the lower limbs as a sensory and motor deficiency has been little studied.

Objectives: To study the dynamics of sensory and motor deficiency against the background of the use of high-frequency low-amplitude TENS (HL TENS) and low-frequency high-amplitude TENS (LH TENS) in the treatment of distal polyneuropathy of the lower limbs in patients with type 2 diabetes mellitus.

Materials and Methods: 122 patients with the distal polyneuropathy of the lower limbs were examined. 40 patients underwent standard medical therapy and, in addition to drug therapy, 41 patients underwent HL TENS $(100 \mathrm{~Hz}-100 \mu$ s - painless sensory response) and 40 patients with LH TENS ( $1 \mathrm{~Hz}-200 \mu \mathrm{s}$-painless motor response). Patients were examined before treatment, immediately after treatment and within 6 months after treatment. Severity of negative sensory symptoms was determined by a 5-point scale with the mean value of temperature, tactile, pain and vibration sensitivity on the two extremities. The paresis of the foot during extension was determined by a 5-point scale. Electromyographic indices of the sural, peroneal and tibial nerves were studied.

Results: TENS enhances the therapeutic effect of standard drug therapy in regression of negative sensory symptoms by 1.3 times and has a prolonged effect that lasts more than 6 months after the end of the course of treatment. In this case, the paresis of the foot against the background of LH TENS regressed with extension by $16.7 \%$ and with flexion by $21.1 \%$ and did not change significantly against the background of HL TENS and drug therapy. Negative sensory symptoms more regressed against background of LH TENS immediately after treatment by $77 \%$ and at the end of the long-term period by 2.2 times compared with HL TENS. The motor deficit regressed against the background of the LH TENS by $16.7 \%$ with the extension of the foot and by $21.1 \%$ with the flexion of the foot and was preserved without significant dynamics for the entire remote period. Against the background of drug therapy and HL TENS, motor deficiency remained without significant dynamics. The dynamics of electromyographic indicators were not significant. However, in some cases there was an improvement in the M-response rate against the background of the use of LH TENS

Conclusion: TENS is a highly effective method for the treatment of distal polyneuropathy of the lower limbs in combination with drug therapy. Negative sensory symptoms, motor deficiency and EMG disturbances regress more against the background of LH TENS with respect to HL TENS.

Keywords: Diabetic neuropathy; Transcutaneous electro neuro stimulation; High-frequency low-amplitude; Low-frequency high-amplitude; Diabetes mellitus

Abbrevations: TENS:Transcutaneous electro neuro stimulation; HL TENS: High-frequency low-amplitude TENS; LH TENS: low-frequency high-amplitude TENS; ENMG: Electroneuromyography 


\section{Introduction}

Since the discovery of the therapeutic effect of transcutaneous electroneurostimulation (TENS), this technique has found its clinical application in practice in the treatment of pain syndrome [1-5]. The collective question of the effectiveness of this method was removed after the birth of the gate control theory of pain in 1965, which gave a pathogenetic explanation of the therapeutic effect of this method curing the pain syndrome [6]. The effectiveness of TENS in the treatment of clinical manifestations of the peripheral nervous system pathology as sensory and motor deficiency has been proved in many works [7-9]. The regression of degenerative changes and the intensification of regenerative processes against the background of TENS has been proven in numerous experimental studies due to the improvement of regional blood circulation $[7,8,10,11]$ antioedematic effect $[12,13]$ strengthening of intra-axonal transport, reinnervation of denervation muscle fibers, anastomosis of affected nerve branches with healthy ones $[14,15]$.

Direct stimulation of the nerve in the treatment of distal polyneuropathy has been used for the first time since 2010 and has been proven by the authors that the direct stimulation of the peroneal and tibial nerves is more effective in treating sensory and motor disorders than in indirect methods of stimulation (stimulation of muscle fibers of $\mathrm{m}$. Tibialis anterior ete $\mathrm{m}$. Triceps surae) [1,2,16-18]. The regression of motor and sensory fibers, depending on the frequency and amplitude of the applied current, has not been sufficiently studied [1, 9,14,19]. In this regard, the purpose of our work was determined: to conduct a comparative analysis between a high-frequency low-amplitude TENS (HL TENS) and a low-frequency high-amplitude TENS (LH TENS) in the treatment of negative and sensory symptoms and motor deficiency in patients with diabetic distal polyneuropathy of the lower extremities with application of innovative scientific and technical complexes and scientific and research technology.

\section{Research Materials}

$\mathrm{N}=122$ (F: 60, M: 62) patients with DM-2 complicated by distal lower extremity polyneuropathy (SLEP) with moderate neuropathic pain syndrome were examined. The severity of the pain syndrome according to the visual analogue scale (VAS) was less than 4 points. The control group: $\mathrm{n}=40$ (F: 20, M: 20) underwent a two-month course of standard drug therapy with adequate oral hypoglycemic therapy [20]. The main group: $\mathrm{n}=82$ (F: 40, M: 42) in addition to the drug therapy conducted in the control group, passed the TENS course. The TENS course started on the second month of drug treatment. Patients of the main group were divided into two subgroups depending on the TENS form: the first subgroup $n=41$ (F: 21, M: 20) completed the course of the LH TENS and the second subgroup $n=41$ (F: 19, M: 22) completed the course of LH TENS.

\section{Methods of Research and Treatment}

The study of negative sensory symptoms was carried out by determining the severity of tactile, temperature, pain, and vibration perception [21]. a) Tactile and protective perception was tested using a monofilament $10 \mathrm{~g}$ by "Semmens-wienstien NCM", series 5,07, USA.

b) Temperature perception was studied with the help of the theotherm "Thio-Therm" by NeueMedizintechnic GMBH, Germany.

c) Pain perception was studied using a special Neuropen and sterile Neurotips by "OwenMuford”, USA.

d) The vibration perception was verified with a graduated tuning fork "Riedel-Siefert" ("Kicher + Wilhelm", Germany) $128 \mathrm{~Hz}$.

e) The research was carried out by determining the zones of decrease in tactile, temperature and pain perception in the examined areas of the skin. The study of the lower extremities was conducted starting from the skin area where the patient well distinguished the contact of the monofilament in the study of tactile perception, clearly distinguished the cold from the warm, when examining the temperature perception, and distinctly differentiated the acute end from the blunt, when examining the pain perception. Errors in the perception of differences in the study of each of these perceptions were taken as a sign of a damage of the perception studied. The severity of each of the disorders of tactile, temperature and pain perception was determined on a 5-point scale, depending on the level of onset of hypoesthesia on the lower limb. The decrease in perception at the level of the distal toe corresponded to 1 point, at the midpoint of the foot - 2 points, at the midpoint of the ankle - 3 points, at the mid-calf level - 4 points and at the knee joint level - 5 points.

In the study of vibration perception, the tuning fork was applied to the bony prominence of the terminal phalanx of the big toe and the medial surface of the calcaneal bones when examining the lower extremities.

The severity of the vibration perception was determined on 8-point scale. On both branches there is a wedge-shaped scale with divisions from 1 to 8 . When you click on the branch, an optical illusion of the intersection of the 2 lines is created. The significance of the results obtained in the study of vibration perception on 8-point scale was translated on a 5-point scale according to (Table 1). The motor deficit was determined by determining the strength of the foot when flexing and extending on a 5-point scale.The electroneuromyography (ENMG) study of the peroneal and tibial nerves was performed with the determination of the nerve motor conduction velocity and the amplitude of the M-response. TENS was performed on the MBN-stimulus apparatus of MBN firm with registration No. FSR 2011/10956Characteristics of the current when conducting HL TENS: monophasic current, rectangular with a frequency of $100 \mathrm{~Hz}$ and a duration of 100 microseconds. Stimulation was intermittent with a stimulation cycle duration of $300 \mathrm{~ms}$. The cycle frequency is $1 \mathrm{~Hz}$. The amplitude was adjusted individually, from 5-50mA to reach painless sensory sensations. 
Stimulation of the peroneal and tibial nerves was carried out under the control of electroneuromyography (ENMG).

Table 1: Translation of the vibration perception thresholds into points for calculating the vibration perception violation by a 5-point scale.

\begin{tabular}{|c|c|c|}
\hline Points & $\begin{array}{c}\text { The threshold of } \\
\text { vibration perceptionat } \\
\text { the base of 1 finger }\end{array}$ & $\begin{array}{c}\text { The threshold ofvibration } \\
\text { perceptionin the medial } \\
\text { malleolus }\end{array}$ \\
\hline 0 & $\geq 6$ & $\geq 6$ \\
\hline 1 & 5 & $\geq 6$ \\
\hline 2 & $1-4$ & 5 \\
\hline 3 & $1-4$ & $1-4$ \\
\hline 4 & 0 & $1-4$ \\
\hline 5 & 0 & 0 \\
\hline
\end{tabular}

The number of sessions was 15 and was performed every other day [1-3].Characteristics of the current for conducting LH amplitude TENS: current monophasic, rectangular with a frequency of $1 \mathrm{~Hz}$ and a duration of 200 microseconds. The amplitude is adjusted individually from 5-50mA to the achievement of painless muscle contraction. Stimulation of the peroneal and tibial nerves under the control of ENMG was carried out. The number of sessions was 15 and was performed every other day [1-3]. Statistical processing of research results was carried out using the application package: Statistica 7.0. Average values of the variational series were determined (arithmetic mean M, standard deviation - $\sigma$ ). A difference method was used to calculate the t-test for the reliability of the difference in the results by Student (p).

\section{Results of the Study \\ Results of the Study of Sensory Deficiency}

The results of the study of negative sensory symptoms in the control group and subgroups of the TENS course are shown in (Table 2) demonstrates that prior to treatment, negative sensory symptoms in the TENS control group and subgroups were pronounced and averaged $2.4 \pm 0.11$ points.As a result of the conducted treatment, there was a significant decrease in the average values of negative sensory symptoms compared to baseline before treatment in the control group by $13.3 \%(\mathrm{p}<0.05)$ and in the subgroups of the HL TENS $22.1 \%(p<0,05)$ and LH TENS at $39.2 \%$ ( $p<0.05$ ).The decrease in negative sensory symptoms achieved after the application of the TENS-LH course exceeded the reduction of negative sensory symptoms as a result of the use of the TENS-HL by $77.2 \%(\mathrm{p}<0.05)$.

Table 2: Dynamics of negative sensory symptoms in different periods of observation.

\begin{tabular}{|c|c|c|c|c|}
\hline \multirow{2}{*}{ Vibration hypoesthesia } & \multirow{2}{*}{ Before treatment } & \multirow{2}{*}{ After treatment } & \multicolumn{2}{|c|}{ Remote period } \\
\hline & & & 2 months & 6 months \\
\hline The control group & $3,1 \pm 0,15$ & $3,1 \pm 0,15^{\prime \prime}$ & $3,0 \pm 0,14^{\prime \prime}$ & $3,0 \pm 0,15^{\prime \prime}$ \\
\hline ВН ТЭНС & $2,9 \pm 0,14$ & $2,8 \pm 0,15^{\prime \prime}$ & $2,9 \pm 0,15^{\prime \prime}$ & $3,0 \pm 0,15^{\prime \prime}$ \\
\hline НВ ТЭНС & $3,1 \pm 0,12$ & $2,6 \pm 0,12 * \#$ & $2,5 \pm 0,12 * \#$ & $2,5 \pm 0,12 * \#$ \\
\hline \multirow[t]{2}{*}{ Thermalhypoesthesia } & \multirow{2}{*}{ Before treatment } & \multirow{2}{*}{ After treatment } & \multicolumn{2}{|c|}{ Remote period } \\
\hline & & & 2 months & 6 months \\
\hline The control group & $2,4 \pm 0,14$ & $2,0 \pm 0,14^{* \prime \prime}$ & $1,7 \pm 0,13^{* \prime \prime}$ & $2,4 \pm 0,14^{\prime \prime}$ \\
\hline HL TENS & $2,5 \pm 0,13$ & $1,8 \pm 0,11^{* \prime \prime}$ & $1,7 \pm 0,11^{* \prime \prime}$ & $2,3 \pm 0,14^{\prime \prime}$ \\
\hline LH TENS & $2,5 \pm 0,12$ & $1,1 \pm 0,09 * \#$ & $0,7 \pm 0,07^{*} \#$ & $1,6 \pm 0,07 * \#$ \\
\hline \multirow[t]{2}{*}{ Tactile hypoesthesia } & \multirow{2}{*}{ Before treatment } & \multirow{2}{*}{ After treatment } & \multicolumn{2}{|c|}{ Remote period } \\
\hline & & & 2 months & 6 months \\
\hline The control group & $2,3 \pm 0,13$ & $1,8 \pm 0,13^{* \prime \prime}$ & $1,6 \pm 0,13^{* \prime \prime}$ & $2,2 \pm 0,13^{\prime \prime}$ \\
\hline HL TENS & $2,2 \pm 0,12$ & $1,5 \pm 0,08^{*} \#^{\prime \prime}$ & $1,3 \pm 0,09 * \#^{\prime \prime}$ & $1,8 \pm 0,09^{*} \#^{\prime \prime}$ \\
\hline LH TENS & $2,1 \pm 0,12$ & $1,2 \pm 0,08^{*} \#$ & $1 \pm 0,07 * \#$ & $1,5 \pm 0,1^{*} \#$ \\
\hline \multirow[t]{2}{*}{ Pain hypoesthesia } & \multirow{2}{*}{ Before treatment } & \multirow{2}{*}{ After treatment } & \multicolumn{2}{|c|}{ Remote period } \\
\hline & & & 2 months & 6 months \\
\hline The control group & $2 \pm 0,13$ & $1,6 \pm 0,12^{* \prime \prime}$ & $1,5 \pm 0,13^{* \prime \prime}$ & $1,9 \pm 0,13^{\prime \prime}$ \\
\hline HL TENS & $1,9 \pm 0,1$ & $1,3 \pm 0,08^{*} \#^{\prime \prime}$ & $1 \pm 0,07^{*} \#^{\prime \prime}$ & $1,6 \pm 0,08 * \#$ \\
\hline LH TENS & $2 \pm 0,12$ & $1,0 \pm 0,07 * \#$ & $0,7 \pm 0,07^{*} \#$ & $1,5 \pm 0,09 * \#$ \\
\hline \multirow[t]{2}{*}{ Mean value of negative sensory symptoms } & \multirow{2}{*}{ Before treatment } & \multirow{2}{*}{ After treatment } & \multicolumn{2}{|c|}{ Remote period } \\
\hline & & & 2 months & 6 months \\
\hline The control group & $2,5 \pm 0,13$ & $2,1 \pm 0,13^{* \prime \prime}$ & $2,0 \pm 0,13^{* \prime \prime}$ & $2,4 \pm 0,13^{\prime \prime}$ \\
\hline HL TENS & $2,4 \pm 0,12$ & $1,9 \pm 0,12^{*} \#^{\prime \prime}$ & $1,7 \pm 0,10^{*} \#^{\prime \prime}$ & $2,2 \pm 0,10^{\prime \prime}$ \\
\hline LH TENS & $2,4 \pm 0,11$ & $1,5 \pm 0,09 * \#$ & $1,2 \pm 0,08^{*} \#$ & $1,8 \pm 0,09 * \#$ \\
\hline
\end{tabular}

Note: $\mathrm{p}<0,05$ - Significant difference between results before rand after treatment; \# - $\mathrm{p}<0,05$ - Significant difference between results of TENS groups and control group; “-p $<0.05$ - Significant difference between results of LH TENS group and results of control and HL TENS groups. 
It is important to note that for the first 2 months of the longterm period, the surface sensitivity indicators continued to improve against the background of LH TENS at $16.9 \%$ ( $p<0.05$ ). After the application of standard drug therapy and against the background of the use of HL TENS, the indices of negative sensory symptoms were preserved without negative dynamics ( $p>0.05)$. At the end of the long-term period- 6 months after treatment - there was an increase in the severity of negative sensory symptoms in all the examined groups. At the same time, negative sensory symptoms in patients with LH TENS were lower than the baseline values before treatment by $26.8 \%(\mathrm{p}<0.05)$.These indices in patients after HL TENS were lower than the previous level before treatment by only $8.4 \%$ ( $(p>0.05)$. In the background of standard drug therapy, the negative sensory symptoms before and after treatment did not differ significantly.
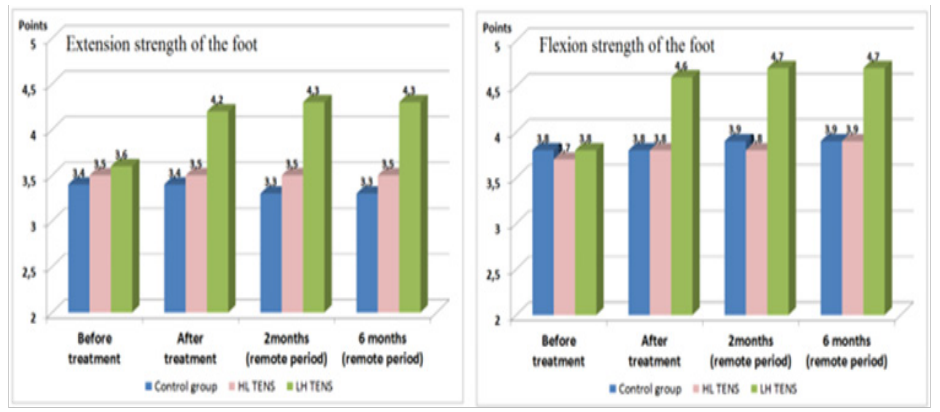

Figure 1: Dynamics of extension and flexion strength of the foot in the treatment during various observation periods.

\section{Results of the Study of Motor Deficiency}

In the study of motor deficiency, the strength of the foot was determined during flexion and extension in patients with foot paresis. The paresis of the foot during extension was revealed in $16.5 \%$ of the examined limbs and when flexing in $11 \%$ of the limbs. The results are shown in (Figure 1) As a result of this study, it was revealed that the improvement of the motor sphere was observed exclusively against the background of LH TENS and was not observed against the background of HL TENS and drug therapy. In this case, the foot strength improved with extension to $16.7 \%$ $(\mathrm{p}<0.05)$ and when flexed to $21.1 \%(\mathrm{p}<0.05)$ and was preserved without negative dynamics for the entire long-term period.

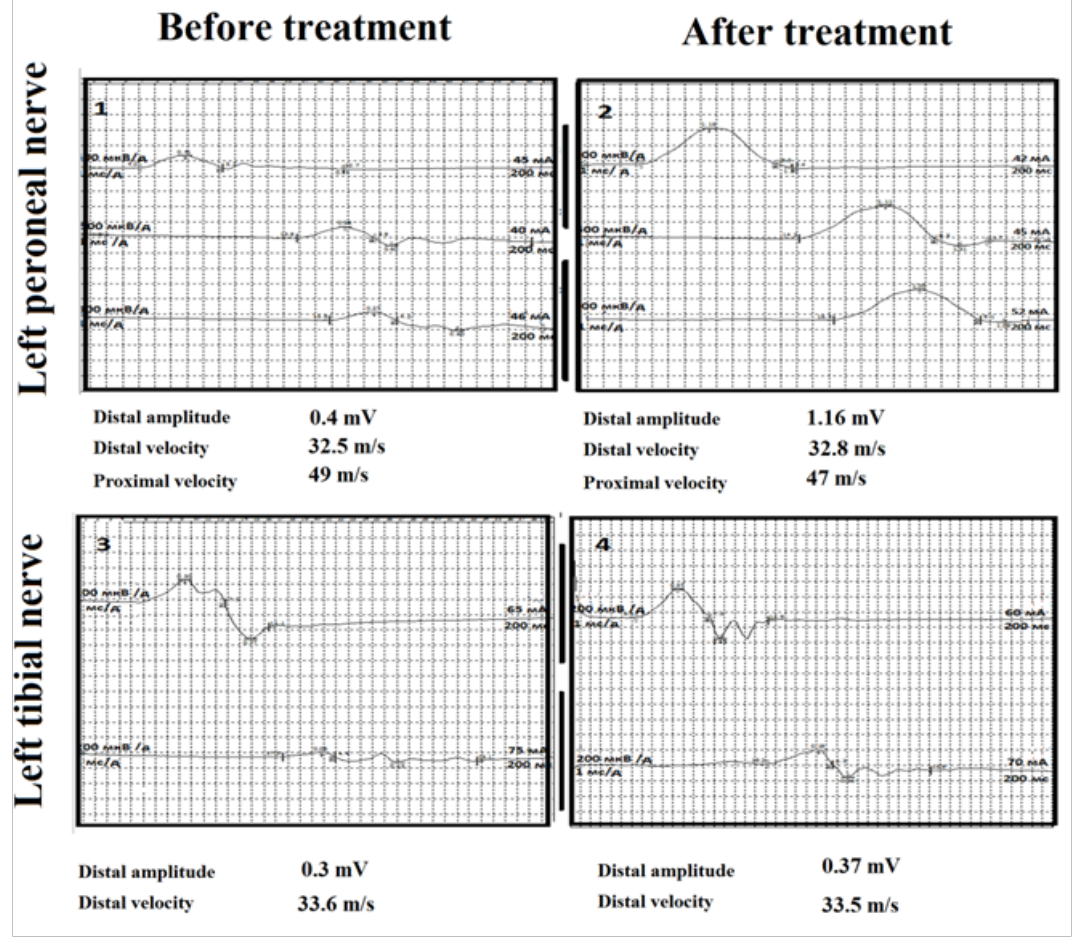

Figure 2: Increase in the amplitude of the M-response of the peroneal and tibial nerves after the application of LH TENS in the treatment of patient with distal polyneuropathy of the lower limbs.

Note: Restoration of the amplitude of the M-response in the study of the peroneal and tibial nerves with the preservation of conduction velocity without significant dynamics. 1 and 3 before treatment. 2 and 4 after treatment. 


\section{Results of Electroneuromyography Examination}

The dynamics of the results of ENMG studies of motor fibers of the peroneal and tibial nerves against the background of the treatment was not reliable. However, in some cases, we observed the restoration of the amplitude of the M-response in the study of motor fibers of the peroneal and tibial nerves only against the background of the use of LH TENS and was not observed after HL TENS and drug therapy, as indicated in (Figure 2).

\section{Conclusion}

The results of application of innovative scientific and technical complexes and scientific and research technology made it possible to prove and scientifically substantiate high efficiency of TENS of various modalities in the treatment of sensory and motor deficiency in patients with diabetic distal polyneuropathy in type 2 diabetes mellitus.At the same time, the TENS enhances effectiveness of standard drug therapy in regression of negative sensory symptoms immediately after the course of treatment by 1.3 times and has a prolonged effect that lasts for 6 months after the end of the course of treatment, which is not observed with the exclusive use of standard drug therapy.LH TENS exceeds the effect of HF TENS in reducing the severity of negative sensory symptoms immediately after treatment by $77 \%$ and 2.2 times in the long-term period.At the same time, the strength of the foot when flexing and extending was regressed only against the background of LH TENS and was not observed against the background of HL TENS and drug therapy with the retention of the motor deficiency regression for the entire long-term period.In some cases, an increase in theamplitude of the M-response was recorded in the study of the peroneal and tibial nerves with the help of ENMG.

\section{References}

1. Al Zamil MKh, Minenko SA, IA Bozhko, LM Kudaeva (2015) Complex application of transcutaneous electro neuro stimulation and acupuncture in the treatment of diabetic distal polyneuropathy of the lower limbs. Clinical neurology, Moscow, p. 14-19.

2. Vance, GT Carol, L Dailey Dana, A Barbara Rakel, K.A Sluka (2014) Using TENS for pain control: the state of the evidence. Pain Manag 4(3): 197209.

3. Josimari M De Santana, Deirdre M Walsh, Carol Vance, Barbara A Rakel, Sluka (2008) Effectiveness of Transcutaneous Electrical Nerve Stimulation for Treatment of Hyperalgesia and Pain. Curr Rheumatol Rep 10(6): 492-499.

4. Karin Pieber, P Karin, H Malvina, T PaternostroSluga (2010) Electrotherapy for the treatment for the painful diabetic neuropathy: a review. J Rehabil Med 42(4): 289-295.

5. Kochetova, MH Al Zamil, LM Kudaeva, IA Minenko, SA Bozhko (2015) Electrotherapy in the treatment of diabetic polyneuropathies.-Historical aspect. Clinical neurology Moscow, p. 27-33.
6. R Melzack, PD Wall (1965) Pain mechanisms: a new theory. Science 150: 971-979

7. Baldwin ERL, Baldwin T, Lancaster J, Mc Neely M, Collins D (2012) Neuromuscular electrical a case-series report. Physiother Can 64(3): 317-324.

8. Broderick BJ, Breathnach O, Condon F, Masterson E, Olaighin G (2013) Haemodynamic performance of neuromuscular electrical stimulation (NMES) during recovery from total hip arthroplasty. J Orthop Surg 8: 3.

9. Chen C, Johnson M, McDonough S, Cramp F (2007) The effect of transcutaneous electrical nerve stimulation on local and distal cutaneous blood flow following a prolonged heat stimulus in healthy subjects. Clin PhysiolFunct Imaging 27(3): 154-161.

10. Liebano RE, Ferreira LM, Sabino Neto M (2003) Experimental model for transcutaneous electrical nerve stimulation on ischemic random skin flap in rats. Acta Cir Bras 18: 54-59.

11. Sandberg ML, Sandberg MK, Dahl J (2007) Blood flow changes in the trapezius muscle and overlying skin following transcutaneous electrical nerve stimulation. Physical Therapy 87(8): 1047-1055

12. Hunckler J, De Mel A (2017) Electrotherapy in wound healing. Journal of Multidisciplinary Healthcare 10: 179-194.

13. Kavak S, Tulgar M, Anlar O (2010) Effects of transcutaneous electrical nerve stimulation on motor and sensorial nerves for diabetic polyneuropathy patients by use of electromyography. Cell Membranes and Free Radical Research 3(2): 112-116.

14. Josimari M De Santana, Deirdre M Walsh, Carol Vance, Barbara A Rakel, Sluka (2008) Effectiveness of Transcutaneous Electrical Nerve Stimulation for Treatment of Hyperalgesia and Pain. CurrRheumatol Rep 10(6): 492-499.

15. Karin Pieber, P Karin, H Malvina, T PaternostroSluga (2010) Electrotherapy for the treatment for the painful diabetic neuropathy: a review. J Rehabil Med 42(4): 289-295.

16. Kochetova, MH Al Zamil, LM Kudaeva, IA Minenko, SA Bozhko (2015) Electrotherapy in the treatment of diabetic polyneuropathies.-Historical aspect. Clinical neurology Moscow, p. 27-33.

17. Polak A, Franek A, Taradaj J (2014) High-voltage pulsed current electrical stimulation in wound treatment. Adv Wound Care (New Rochelle) 3(2): 104-117.

18. Roberta AC Folha, Carlos E Pinfildi, Richard E. Liebano (2015) Can transcutaneous electrical nerve stimulation improve achilles tendon healing in rats? Braz J Phys Ther (6): 433-440.

19. Moharic M, Burger H (2010) Effect of transcutaneous electrical nerve stimulation on sensation thresholds in patients with painful diabetic neuropathy: an observational study. Int J Rehabil Res 33(3): 211-217.

20. Al-Zamil MKh (2015) The Russian Federation. A method for the treatment of diabetic distal sensorimotor polyneuropathies. The Russian Federation.

21. Kulikova NG, Aleksandrovich GA (2010) Modern medical and social aspects of diabetology. Bulletin of the North Caucasus No. 1: 45-50. 
(c) (i) This work is licensed under Creative

Submission Link: https://biomedres.us/submit-manuscript.php

Assets of Publishing with us
- Global archiving of articles
RESEARCHES

